\title{
Hybrides Projektmanagement
}

\author{
Arnd Albrecht ${ }^{1,2}$ (D) Evelyn Albrecht ${ }^{3}$
}

Online publiziert: 2. Februar 2021

(c) Der/die Autor(en) 2021

\section{Zusammenfassung}

Exzellentes Projektmanagement ist der kritische Erfolgsfaktor für alle Produktentwicklungen und Serviceleistungen in der Wirtschaft. Effektivität und Effizienz sind die Kernparameter, um die Zielerreichung erfolgreich zu gestalten. Globale Wertschöpfungsketten und Beteiligung vieler Stakeholder sowie regulatorische Implikationen führen zu einer immer komplexeren Projektsteuerung und -ausführung in der klassischen sequenziellen Abwicklung. Schwerer kalkulierbare Zeitaufwände und Kosten sowie die volatile Erwartungshaltung des Kunden lassen Projekte risikoreicher werden. Im Zuge der Arbeitswelt 4.0, wo nicht nur Automatisierungen und Künstliche Intelligenz (KI) eine Rolle spielen, haben sich neue agile Projektformen wie Scrum-Management etabliert. Es hat sich allerdings gezeigt, dass reine agile Formen sowohl organisationsbedingt als auch im Zusammenspiel vieler heterogener Partner nicht immer erfolgreich sind. Daraus haben sich Mischformen, sogenannter hybride Gebilde entwickelt, die im Projektmanagement als state of the art gesehen werden. Voraussetzung zur erfolgreichen Umsetzung ist eine hohe Professionalität aller Beteiligten und die Kenntnisse von klassischen und agilen Projektmanagement Formen. Neue Anforderungsprofile für Beteiligte im hybriden Projektmanagement werden diskutiert.

Schlüsselwörter Agiles Projektmanagement · Arbeitswelt 4.0 • Blended Learning · Daily Meetings · Future Skills · Hybrides Projektmanagement - Innovative Organisation - Klassisches Projektmanagement - Scrum - Scrum-Master · Sprint · V-Projekt-Model · VUKA-Welt · Wasserfall-Projektstruktur

\section{Hybrid project management}

\begin{abstract}
Excellence is the critical success factor in project management for product development and service industry. Effectivity and efficiency are the key performance indicators which drive success in all projects. The complexity of project execution is increased by globalized supply chains, the involvement of multiple stakeholder groups, and by the ongoing change of legal regulations. Critical pathways and time constraints as well as increased customer expectations have meant that projects have become more risky. Combined with the fourth industrial revolution, Work 4.0, where AI (Artificial Intelligence) and automation play a more important role, new agile project methods such as Scrum and Kanban have been developed. Both classical as well as pure agile project management formats are not always successful if many heterogenous partners are involved. Thus, it has became relevant for merged models to lead to a new form of project management called hybrid project management. Prerequisites for such a management form is a high level of professionalism and competence from all those involved and a knowledge of both, classical and agile project management. New competencies and job requirements for hybrid project manager are discussed in this article.
\end{abstract}

Keywords Work 4.0 Hybrid project management - Scrum Master · Agile competencies · Waterfall- and V-project model

Arnd Albrecht

arnd.albrecht@munich-business-school.de

1 Munich Business Coaching Institute (MBCI), Ohmstraße 13, 80802 München, Deutschland
2 Munich Business School, Elsenheimerstraße 61, 80687 München, Deutschland

3 FH Südwestfalen, Haldener Str. 182, 58095 Hagen, Deutschland 
Abb. 1 Typische Komponenten des klassischen Projektmanagements mit der WasserfallMethode (links) und dem agilen Projektmanagement (rechts) mit dem Scrum-Format (eigene Darstellung)

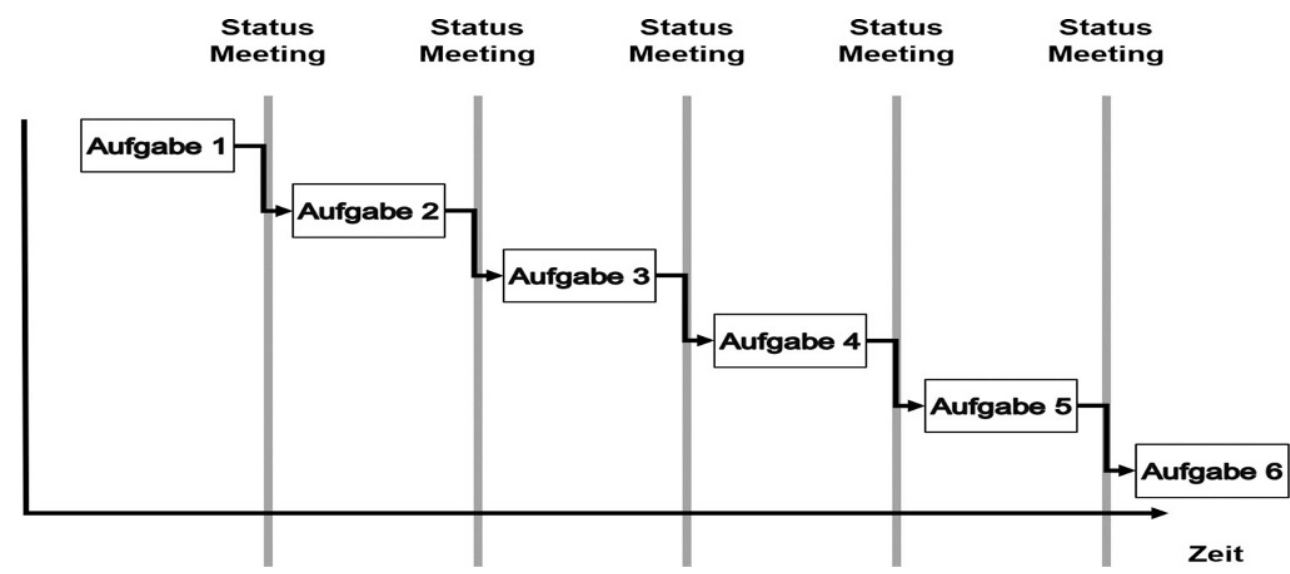

\section{Gescheiterte Projekte und unzureichende Umsetzung}

Fast 10 Jahre nach dem geplanten Fertigstellungstermin wurde der Großraumflughafen Berlin 2020 eröffnet. Tatsächlich stößt man bei der Untersuchung auf Fehler des klassischen Projektmanagements (Delius 2016). Man braucht nicht in die Detailanalyse für die Gründe von gescheiterten Projektmanagement zu gehen, da sie offensichtlich und schon lange bekannt sind (Avots 1969; Hajikazemi et al. 2017). Die Kardinalfehler sind enormer Zeitdruck, Wettbewerbsverzerrung, Arbeiten mit falschen Ausgangsdaten, überzogene Anforderungen, überhastete Umsetzung, fehlende Kooperation der beteiligten Stakeholder und verarmtes Risikomanagement (Arashpour et al. 2016). Die Frage, die sich stellt, gibt es Alternativen zum klassischen Projektmanagement, die Fehler reduzieren und besser Planungssicherheit versprechen. Gibt es gerade vor dem Hintergrund eines sich stark verändernden und wenig vorhersagbarem Umfeld, einer sogenannten VUKA-Welt, mit ihren widrigen Parametern der Volatilität, Unsicherheit, Komplexität und Ambiguität, eine Möglichkeit, flexibler und dadurch besser angepasst mit Projekten umzugehen?

\section{Gegenüberstellung von klassischem versus agilem Projektmanagement}

Ein Hauptunterscheidungsmerkmal beider Systeme ist einerseits im traditionellen Projektmanagement ein direktiver unipolarer Prozess, streng nach einer Richtung verlaufend, andererseits im agilen Projektmanagement ein Schleifenprozess, der durch mehrfache Rückkopplung funktioniert (Abb. 1).

Dabei ist das Merkmal für das klassische Projektmanagement ein Wasserfall-System (Abb. 1) oder etwas weiterentwickelte Verfahren wie das V-Modell, das noch mehr Rückkopplungen hat. Für agiles Projektmanagement ist die
Portionierung in kleinere Einheiten typisch. So werden bestimmte Entwicklungsphasen in dem als Scrum bezeichneten Modell in unabhängig Projektmodule unterteilt, damit ist eine höhere Flexibilität gewährleistet (Fowler 2019). Vergleichbar mit einem Computer-Prozessor werden in sogenannten Sprints diese inhaltlich frei bearbeitet, nur die Produktanforderung und der Zeitrahmen wird vorgegeben und durch den Scrum-Master überwacht (Tab. 1).

Jedes System hat Vor- und Nachteile und man kann generell nicht von vornherein sagen, ob das eine besser ist als das andere. Je nach Organisation, Größe und Komplexität des Projekts, involvierten Stakeholder, Erfahrung des Teamleiters sowie die Erfahrung und Professionalität der Teammitglieder ist das eine dem anderen überlegen (Copola Azenha et al. 2020).

Agiles Projektmanagement hat auch seine Schattenseiten, da bei zu hoher Volatilität die Effektivität leidet. Das Projetteam verliert das Ziel bei zu großer Detailierung inhaltlich komplett aus den Augen oder Spezialisierung führen zu nicht balancierten Ergebnissen bei der Produktentstehung (McConnell 1998).

Darüber hinaus hat sich gezeigt, dass in Teams, die zu stark auf sich fixiert sind, ohne die Involvierung Dritter die Interpretation von positiven Ergebnissen eher verfolgt werden, ohne sie in Frage zu stellen. So werden sie leichter zu möglichen Fehleinschätzungen verleitet (Mojzisch et al. 2014). Bei komplexeren Teamaufgaben, wie beispielsweise in der Gesundheitsindustrie, überwiegt trotzdem der Gesamtnutzen des Teams (Schmutz et al. 2019). Bei agilen Projektmanagementformen kontrolliert zwar der ScrumMaster den Ablauf, tritt aber nicht als Sparringpartner auf und greift dadurch nicht inhaltlich in die Weiterentwicklung bzw. Lösung von Aufgaben ein. Je stärker nun agile Formen in traditionellen Projektmanagement Prozessstrukturen laufen desto gerichteter der Output und die Überwachung, was zum neuen Modell des hybriden Projektmanagement führt. 
Tab. 1 Gegenüberstellungen der Hauptmerkmale von klassischem versus agilem Projektmanagement

\begin{tabular}{lll}
\hline $\begin{array}{l}\text { Unterscheidungs- } \\
\text { merkmal }\end{array}$ & Klassisches Projektmanagement & Agiles Projektmanagement \\
\hline $\begin{array}{l}\text { Haupt- } \\
\text { Charakteristika }\end{array}$ & Wasserfall-System & Sprints (Scrum) \\
& Sequenzierung & Daily Meeting \\
Vorteile & WBS (Work-Breakdown Structure) & Verteilung der Arbeitsaufgaben über Kanban \\
& Klare Übersicht von Abhängigkeiten & Projekt-Entwicklungszeit kurz \\
& Systematischer Ansatz & Große Projekttransparenz \\
& Klare Rollen \& Verantwortlich-keiten (auch bei Übergabe) & Probleme werden schneller angezeigt \\
& Prozessschritte einfacher zu errechnen & Flexibles Eingreifen an jeder Stelle möglich \\
& Budget und Arbeitskraft-Allokation einfacher & Motivierend durch transparente Beteiligung aller \\
Nachteile & Prozessstabilität & Hoher Kommunikationsfluss \\
& Ressourcenverknappung einzelner Arbeitspakete führt zur & Permanente Änderungen stören Gesamtablauf \\
& Verzögerung & \\
& Kritische Pfade haben keine Alternativen & Nächste Schritte oft nicht vorhersagbar, unübersichtlich \\
& Routinen führen zur Monotonie (Attraktivitätsminderung) & Experten und Teammitglieder mit Erfahrung gebraucht \\
& Fehler werden zu spät entdeckt - kein lernender Organismus & Ständiges updating erfordert hohe Disziplin \& Committ- \\
& & ment \\
Anwendung & Großangelegte Projekte & Kleine, überschaubare Projekte \\
& Langfristige Planung & Spontanität erforderlich \\
& Komplexe Projekte & Spezifischer Fragestellung \\
& Involvierung vieler Stakeholder & Experten und wenige Parteien
\end{tabular}

\section{Hybrides Projektmanagement}

Die Kombination von alten und neuen Systemen findet oft statt, und ist mehr als die Summe der Einzelteile. Für interpersonelle Aktivitäten handelt es sich vielmehr um komplementär ergänzende Teile, die aufeinander angewiesen sind, und dadurch einen synergistischen Mehrwert erzeugen und sich besser in vorhandenen Organisationsstrukturen und -denkweisen einfügen (Copola Azenha et al. 2020).

Um die Implikationen beim komplexen Projektmanagement einzuordnen und den Begriff besser zu verstehen seien zwei ,hybride“ Beispiele aus dem Business dargestellt:

Hybride Lernformen, sogenanntes Blended Learning, bieten relativ freie Gestaltung von Trainingseinheiten an. Diese werden größtenteils virtuell durchgeführt, womit sowohl Reisezeit und -kosten reduziert als auch durch moderne Medien interessant gestaltet werden können. Diese Trainingsphase wird aber mit punktuellen physischen Face-to-Face-Meetings durchsetzt, um das Erlernte nach zeitlichen Abständen mit den anderen Teilnehmern zu diskutieren und Methoden/Techniken anzuwenden (Abb. 2). Diese persönlichen Kontakte sind wichtig, um Netzwerk und Vertrauen weiter aufzubauen (Albrecht 2016). Auch diese Form des Wissenstransfer findet beim hybriden Projektmanagement statt. Neben den daily meetings, die online sind, sollten sich aber bei Großprojekten in regelmäßigen Abständen Mitarbeitende mit den Teammitgliedern anderer Bereiche persönlich treffen, um methodische und inhaltliche Lösungswege auszutauschen.
Leider ist die Verwendung des Begriffs ,hybrid“ durch den Schulunterricht in Deutschland zu Zeiten von Corona irreführend und falsch verwendet. Hier handelt es sich exemplarisch nämlich gerade nicht um eine Kombination von zwei aufeinander abgestimmten Systemen, sondern nur um zwei nebeneinandergestellte Systeme, die wenig bis gar nicht harmonisieren. Also ein anschauliches Negativbeispiel wie es nicht sein sollte, was $\mathrm{zu}$ den wenig effizienten und effektiven Resultaten führt. Was bleibt, ist eine Kombination aus unzureichendem partiell vom Lehrkörper durchgeführten Schulunterricht und home-schoolings durch fachlich-didaktisch und mental überforderte Eltern (Anmerkung der Autoren). Hinweise für durchaus erfolgreichen Einsatz von Kombinationen klassischer und agiler Methode analog dem hybriden Projektmanagement gab es schon vor der COVID19-Krise und kann auch in Schulsystemen angewendet werden (Baham 2019).

Ein weiteres Beispiel für eine hybride Form, hat John Kotter (2012) auf Organisationsebene hinsichtlich permanenten Change-Managements skizziert. Kotter beschreibt zwei Betriebssysteme. Die Aufbau- und Ablauforganisation stellt das erste Betriebssystem dar, das der Organisation durch hierarchische Systeme, Prozesse sowie klare Rollen und Verantwortlichkeiten für das operative Geschäft Halt gibt. Das zweite überlagerte Betriebssystem bedient sich aller Hierarchiestufen, Fachbereichen, Kompetenzbereichen usw. Einzelne Personen unterschiedlicher Organisationsebenen agieren nun in dieser neuen Dimension und können innovativ Veränderungen bewerkstelligen, da sie in dieser 
Abb. 2 Blended Learning als hybride Managementform des Wissenstransfers (eigene Darstellung)
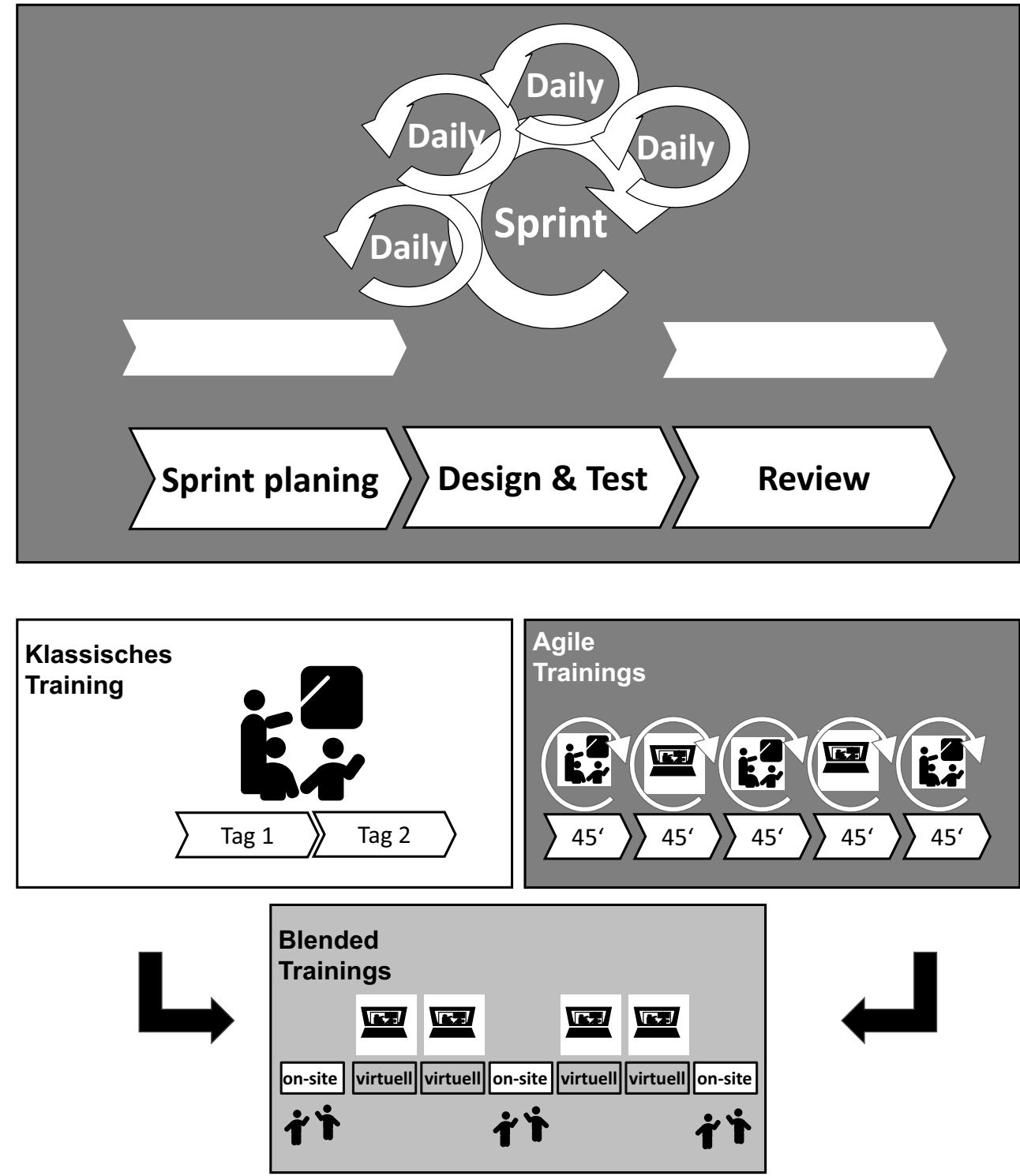

Abb. 3 Agile Organisationen mit zwei Betriebssystemen nach Kotter (2012) als hybride Change-Managementform (eigene Darstellung)
Funktion nicht an ihr fachbezogenes und disziplinarisches System gebunden sind (Abb. 3). Diese überlagerte Organisationsform findet bei hybriden Projektmanagementorganisationen Anwendung, indem beispielsweise ein Entwicklungsprojekt parallel von einer Task-Force begleitet wird, die sich aus verschiedenen Fach- und Funktionsbereichen, Technikern und Theoretiker sowie Strategen und Umsetzern zusammensetzt. Im Gegensatz zur Holokratie, wo das alte System komplett aufgelöst wird und die Mitarbeitenden in neuen Aktionskreisen arbeiten, bleibt die alte Struktur hier erhalten, was den Vorteil hat, dass in kritischen Situationen ein klarer Bezugspunkt vorhanden ist (Bernstein et al. 2016).

Hybrides Management ist in der Praxis stark prozessual chronologisch geprägt, in der unterschiedliche Standards zum Tragen kommen (Timinger 2017). Der Rahmen, in dem ein Projekt weiterentwickelt wird, folgt einer strengen Rei- henfolge. Innerhalb dieses strengen Korsetts wird allerdings eine hohe Flexibilität - vor allem in der Umsetzungsphase - gewährt. Besonders ist die inkrementelle Umsetzung durch starke iterative Prozesse und durch Time-Boxing verstärkt, durch diese sogenanntes „Sprints“ werden Projekte schneller und trotzdem innovativer umgesetzt.

Es gibt bei hybriden Systemen mannigfaltige Typen, von denen ein Prototyp in Abb. 4 repräsentativ dargestellt wurde. Manche Autoren unterscheiden zwar drei Grundvarianten (Möller 2020), tatsächlich sind durch die Kombination von agilen Methoden basierend auf der klassischen Projektmanagementstruktur sehr viele Ausführungen des hybriden Projektmanagements denkbar. Das ist vergleichbar mit dem Chassis (Fahrzeuggestell), auf dem dann eine jeweils veränderbare Fahrzeugkarosserie mit verschiedenen Funktionen aufgebaut wird. 
Abb. 4 Hybrides Projektmanagement als Synthese von klassischen und agilen Projektmanagementmethoden (eigene Darstellung)

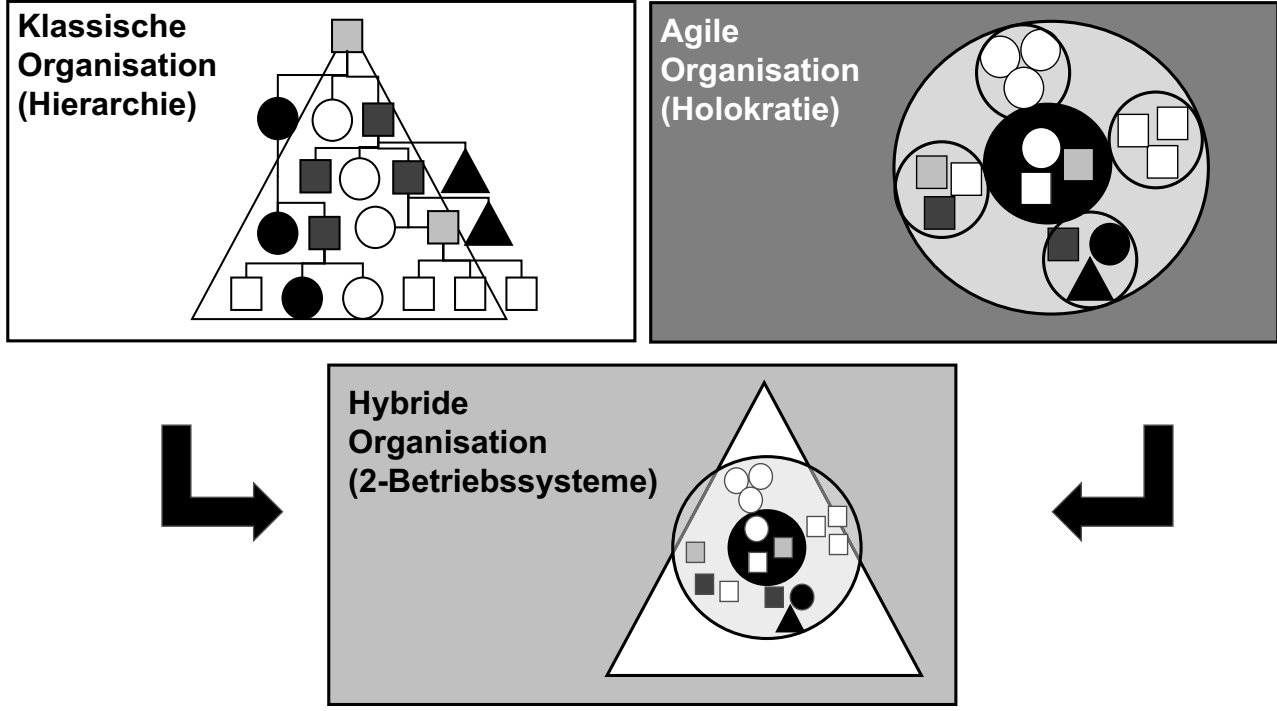

So stellen agile Methoden das Design der „Fahrzeugkarosserie“ und die technische Ausstattung des „Interieurs“ dar. Typische Element der Kommunikation, Priorisierung und Aufgabenverteilung sowie Problemlösungstechniken sind beispielsweise das agile Product Backlog (Ranking neuer oder neuangewandter Produkteigenschaften), die Daily Stand-up Meetings (täglich durchgeführte Abstimmungstreffen - meist virtuell), das Kanban (System der transparenten und agilen Verteilung von Aufgaben), das Time-Boxing (kreativ und effiziente Ideen- und Lösungsfindung unter Zeitdruck), das 360-Grad-Feeback und die Reviews mit Kunden und anderen Projekt-Stakeholder, Kreative Schaffensphasen zur Ideengenerierung (Brainstorming, Pyramidal Thinking/Lego Serious Play ${ }^{\circledR}$ usw.). Wenig ist bisher darüber geforscht und publiziert worden, inwiefern das hybride Projektmanagement den zwei Ursprungsformen überlegen ist (Gemino et al. 2020). Allerdings wird es auch zukünftig schwierig sein gesicherte Aussagen zu machen, da diese drei Formen ja nicht ganz überschneidungsfrei sind und die agilen Elemente recht prozess- und ergebnisumsetzungsbestimmend sind.

\section{Anforderungen durch neue Projektmanagement Formen}

Vor dem Hintergrund der globalen und virtuellen arbeitenden Teams wird das Projektmanagement noch stärker im Fokus stehen, produktiv zu arbeiten (Kankanhalli et al. 2006). Durch die Stärkung der virtuellen Arbeitsformen und Techniken - nicht zuletzt durch COVID19 bedingt - ist die Agilität auch von herkömmlichen klassischen Projekten schon verändert worden. Aufgrund der weiterhin steigenden Komplexität wird eine Grundstruktur notwendig sein, um bei einem vielschichtigen Co-Working-Environment, also viele verschiedene Organisationseinheiten, Orientierungspunkte zu geben (Wysocki 2019). Auch die Anforderungen an das Kompetenzprofil eines hybriden Projektleiters wird nicht mehr nur fachseitig ausgerichtet sein, sondern wird auch Wissen und Erfahrung aus dem Business-Bereich benötigen, in dem er operiert (Ko und Kirsch 2017). Innerhalb dieses Grundrasters wird noch stärker agil gearbeitet werden müssen, um mit der starken volatilen Umgebung Schritt zu halten. Das ist bei kritischen Situationen innerhalb von Projekten zu beobachten, sowohl hinsichtlich Risikomanagements als auch mit dem persönlichen Umgang mit Stress. Dabei kann sich der Mensch zwar neuen methodischen und situativen Herausforderungen anpassen. Allerdings überfordert das Auftreten immer komplexerer kritischer Situationen in einer höheren Frequenz die Stress-Coping Mechanismen (Hillert et al. 2020). Nicht nur neue Methodenkompetenz, wie hybrides Projektmanagement, sondern auch die einzelnen Soft Skills und Fachkompetenzen, also sowohl technischer, interpersoneller als auch intrapersoneller Führung und Lenkung, werden stärker gebraucht als in herkömmlichen Projekten (Müller et al. 2020). Das wiederum erfordert nicht nur eine höhere Professionalität der Projektleiter, sondern aller beteiligten Projektteammitglieder.

\section{Ausblick}

Es besteht kein Zweifel, dass das Projektmanagement sich noch stärker wandeln wird und viele neue Systemkomponenten sowie Methoden und Schnittstellen zur Arbeitswelt 4.0 haben muss (Albrecht 2020). Klassisches Projektmanagement ist bei der immer höheren Taktzahl und Komplexität nicht mehr allein ausreichend. Agile (Projekt)Organisationsformen, flexible Kommunikationsformen 
auch mit KI werden notwendig, was man unter dem Begriff HI (Hybride Intelligence) versteht (Dellermann et al. 2019). Vor allem neue Kompetenzprofile für Projektsteuerung und -umsetzung müssen bei der Aus- und Fortbildung von Projektmanagern und Projektteams stärker berücksichtigt werden (Joiner 2019). Dass es weiterhin zur Verzögerung von Projekten aus politischen Gründen, Korruption und Neuerungen von Gesetzesauflagen kommen kann, ist nicht $\mathrm{zu}$ vermeiden. Allerdings sollten projektmanagementbedingte Verzögerungen der Vergangenheit angehören. Mit hybridem Projektmanagement ist eine Möglichkeit geschaffen, effektiver und effizienter Projekte zu steuern und umzusetzen.

Funding Open Access funding enabled and organized by Projekt DEAL.

Open Access Dieser Artikel wird unter der Creative Commons Namensnennung 4.0 International Lizenz veröffentlicht, welche die Nutzung, Vervielfältigung, Bearbeitung, Verbreitung und Wiedergabe in jeglichem Medium und Format erlaubt, sofern Sie den/die ursprünglichen Autor(en) und die Quelle ordnungsgemäß nennen, einen Link zur Creative Commons Lizenz beifügen und angeben, ob Änderungen vorgenommen wurden.

Die in diesem Artikel enthaltenen Bilder und sonstiges Drittmaterial unterliegen ebenfalls der genannten Creative Commons Lizenz, sofern sich aus der Abbildungslegende nichts anderes ergibt. Sofern das betreffende Material nicht unter der genannten Creative Commons Lizenz steht und die betreffende Handlung nicht nach gesetzlichen Vorschriften erlaubt ist, ist für die oben aufgeführten Weiterverwendungen des Materials die Einwilligung des jeweiligen Rechteinhabers einzuholen.

Weitere Details zur Lizenz entnehmen Sie bitte der Lizenzinformation auf http://creativecommons.org/licenses/by/4.0/deed.de.

\section{Literatur}

Albrecht, A. (2016). Virtuelles Führen als kritischer Erfolgsfaktor in der neuen Arbeitswelt. PERSONALquarterly, 16(1), 17-22.

Albrecht, A. (2020). Arbeitswelt 4.0. In L. v. Rosenstiel, E. Regnet \& M.E. Domsch (Hrsg.), Führung von Mitarbeitern: Handbuch für erfolgreiches Personalmanagement (8. Aufl. S. 733-745). Stuttgart: Schäffer-Poeschel.

Arashpour, M., Wakefield, R., Lee, E.W.M., Chan, R., \& Hosseini, M.R. (2016). Analysis of interacting uncertainties in on-site and off-site activities: implications for hybrid construction. International Journal of Project Management, 34(7), 1393-1402.

Avots, I. (1969). Why does project management fail? California Management Review, 12(1), 77-82.

Baham, C. (2019). Teaching tip: implementing Scrum wholesale in the classroom. Journal of Information Systems Education, 30(3), 141-159.

Bernstein, E., Bunch, J., Canner, N., \& Lee, M. (2016). Beyond the holacracy hype. Harvard Business Review, 94(7/8), 38-49.

Copola Azenha, F., Aparecida Reis, D., \& Leme Fleury, A. (2020). The role and characteristics of hybrid approaches to project management in the development of technology-based products and services. Project Management Journal. https://doi.org/10.1177/ 8756972820956884

Delius, M. (2016). Drucksache 17/3000, 14.06.2016. Bericht des 1 . Untersuchungsausschusses des Abgeordnetenhauses von Berlin 17. Wahlperiode - zur Aufklärung der Ursachen, Konsequenzen und Verantwortung für die Kosten- und Terminüberschreitungen des im Bau befindlichen Flughafens Berlin Brandenburg Willy Brandt (BER)

Dellermann, D., Ebel, P., Söllner, M., \& Leimeister, J. M. (2019). Hybrid intelligence. Business \& Information Systems Engineering, 61(5), 637-643.

Fowler, F.M. (2019). Navigating hybrid Scrum environments: understanding the essentials, avoiding the pitfalls (S. 13-22). New York: Apress Media.

Gemino, A., Horner Reich, B., \& Serrador, P. M. (2020). Agile, traditional, and hybrid approaches to project success: is hybrid a poor second choice? Project Management Journal. https://doi.org/10. $1177 / 8756972820973082$.

Hajikazemi, S., Andersen, B., \& Krane, H. P. (2017). A review on possible approaches for detecting early warning signs in projects. Project Management Journal, 44(5), 55-69.

Hillert, A., Albrecht, A., \& Voderholzer, U. (2020). The burnout phenomenon: a résumé after more than 15,000 scientific publications. Frontiers in psychiatry, 11, 519237.

Joiner, B. (2019). Leadership agility for organizational agility. Journal of Creating Value, 5(2), 139-149.

Kankanhalli, A., Tan, B.C. Y., \& Kwok-Kee, W.E. I. (2006). Conflict and performance in global virtual teams. Journal of Management Information Systems, 23(3), 237-273.

Ko, D.-G., \& Kirsch, L. J. (2017). The hybrid IT project manager: one foot each in the IT and business domains. International Journal of Project Management, 35(3), 307-319.

Kotter, J.P. (2012). ACCELERATE! (cover story). Harvard Business Review, 90(11), 44-58.

McConnell, S. (1998). Software project survival guide: how to be sure your first important project isn't your last. Washington: Microsoft Press.

Mojzisch, A., Kerschreiter, R., Faulmüller, N., Vogelgesang, F., \& Schulz-Hardt, S. (2014). The consistency principle in interpersonal communication: consequences of preference confirmation and disconfirmation in collective decision-making. Journal of Personality and Social Psychology, 106(6), 961-977.

Möller, T. (2020). Projektleiter/in Pragmatischer Leitfaden für klassisches, agiles und hybrides Projektmanagement mit Lernhinweisen für die Zertifikate IPMA / GPM Basis- und Level D-Zertifikat sowie Scrum PSM I (2. Aufl.). (S. 96-93). Ganderkesee: con-thor.

Müller, A., Graumann, M., \& Weiß, H.-J. (2020). Innovationen für eine digitale Wirtschaft - Wie Unternehmen den Wandel meistern. Wiesbaden: Springer Gabler.

Schmutz, J.B., Meier, L.L., \& Manser, T. (2019). How effective is teamwork really? The relationship between teamwork and performance in healthcare teams: a systematic review and meta-analysis. Britisch Medical Journal open, 9(9), e28280. https://doi.org/ 10.1136/bmjopen-2018-028280.

Timinger, H. (2017). Modernes Projektmanagement: Mit traditionellem, agilem und hybridem Vorgehen zum Erfolg (1. Aufl.). Weinheim: Wiley-VCH.

Wysocki, R. K. (2019). Effective project management traditional, agile, extreme, hybrid (S. 335-380). Indianapolis: John Wiley \& Sons.

Professor Dr. Arnd Albrecht ist Akademischer Direktor an der Munich Business School und Leiter des MBCI - Munich Business Coaching Institute. Er lehrt Human Ressource Management, Responsible Leadership und Internationales Management. Seine Forschungsschwerpunkte sind Führung, neue Organisationsformen und Health Care Management sowie die Untersuchung über die Interaktion von Organisationen und Mitarbeitenden hinsichtlich deren Entwicklung und Führungsstilen. Neben seiner Professur berät und trainiert er Führungskräfte hinsichtlich Leadership und interkulturellen Kompetenzen und bildet Business Coaches aus. 
Prof. Dr. Evelyn Albrecht ist Professor an der Fachhochschule Südwestfalen und lehrt im Fachbereich Technische Betriebswirtschaft, Unternehmensführung, Unternehmensethik und Projektmanagement. Ihr Forschungsschwerpunkt hat sie auf künstliche Intelligenz und agile Führungs- und Projektmanagementmodelle in Unternehmen gerichtet. Sie ist GPM/IPMA-Assessor und hat als zertifizierte Trainerin die weltweite Einführung der neuen Qualitätsstandards im Projektmanagement des GPM/IPMA-unterstützt, der neben dem amerikanischen PMI die führende Zertifizierungsreferenz ist. 